\title{
Population Viability in Three Trophic-level Food Chains
}

\author{
Noël BONNEUIL \\ Institut national des études démographiques, \\ 133, bld Davout, 75980, Paris cedex 20, France, \\ and École des hautes études en sciences sociales \\ and Patrick Saint-PIERRE, \\ Université de Paris Dauphine, \\ place du Maréchal de Lattre de Tassigny, \\ 75016, Paris, France
}

1

\footnotetext{
${ }^{1}$ corresponding author: Noël Bonneuil: Institut national des études démographiques, 133, bld Davout, 75980, Paris cedex 20, France, tel: 33.1.56.06.21.38, fax: 33.1.56.06.21.99, e:mail: bonneuil@ined.fr.

Acknowledgements: We thank Christine CHOIRAT and Raffaelo SERI for early helpful discussions.
} 


\begin{abstract}
The perpetuation of three-trophic level ecosystems where the three species exhibit unpredictable time-varying survival strategies is best described by a specific set, the viability kernel, gathering all states from which there exists at least one trajectory safeguarding each species over a given density threshold. The strategies permitting this property are delineated and called viable strategies. All solutions starting outside the viability kernel lead to extinction.

Viable strategies vary frequently when the super-predator is abundant, making co-existence more fragile. They still vary, but less, when the super-predator is in low density, implying that coexistence is obtained with less variation of the interactions.

The study of the dependence of the viability kernel on the admissible sets of strategies shows the necessity for the capture rates of predators and super-predators to repeatedly take sufficiently low values.
\end{abstract}

keywords: Trophic food chains — Dynamical systems - Viability theory - Natural selection

\title{
1 Introduction
}

The assembly and persistence in three trophic-level food chains has been an important theme in population dynamics (Freedman and Waltman, 1977, 1984; Gard and Hallam, 1979; Gard, 1980; Freedman and So, 1985; ElOwaidy and Ammar, 1986; Hofbauer and Sigmund, 1988, 1998; Husson and Law, 1992; Law and Blackford, 1992). Interactions within food chains 
are commonly represented by specific functions, deterministic or stochastic, yielding specific interesting asymptotic behaviors, revealed through attractors and their separatrices (Boer, Kooi and Kooijman, 2001). These studies generally show that persistence in these models is obtained through oscillations (Holling, 1965; Muratori and Rinaldi, 1992) or that such models can display bifurcations and chaotic dynamics (Hastings and Powell, 1991; Scheffer, 1991; Wilder et al, 1994; Kuznetsov and Rinaldi, 1996). Morin and Lawler (1995) note that the variety of dynamic behaviors implied by the models depends on the details of the interactions.

As pointed out by Law and Morton (1996: 763), "it is not clear that local asymptotic stability is an appropriate condition for coexistence, for species may coexist without tending to an equilibrium point" (Williamson, 1987; Hastings, 1988); and the "dynamics close to an interior equilibrium point are not the main issue for coexistence". Pianka (1978) observed that the theory of prey-predator interactions could be improved substantially by treating coefficients involved in Lotka-Volterra models "as variables in both ecological and evolutionary time" (p. 199). He mentioned stress as a source of variation, heterogeneity of the environment, he finally emphasized how much the fitness of an organism can "strongly be influenced by the degree of developmental flexibility of a given phenotype trait" (p. 165). Even in laboratory settings, arthropod studies of predator-prey interactions describe either sustained oscillations or irregular fluctuations in population size over time (Huffaker, 1958; Mitchell, Arthur and Farrow, 1992; Pimentel, Nagel and Madden, 1963). Mittelbach et al (1995: 2347) acknowledged the fact that interactions within most ecological communities are often poorly known. 
Wilbur (1972) pointed out the inadequacies of classical models of laboratory ecologists at reflecting trajectories of communities in a changing environment.

In a dynamical game, interactions are no longer given, but appear as strategies. Coevolution was presented as such a game, where each individual was thought to adopt a "fitness-maximizing strategy" (Brown and Vincent, 1985). A species can then be identified to its strategy (Abrams, 1987), or else by a whole strategy set, benefiting to individuals who are not evolutionary identical (Brown and Vincent, 1992). The latter interpretation will prevail in this article.

Brown and Vincent (1992) pointed out that the fitness of an individual in a community is bounded by evolutionary constraints. Strategies are allowed to vary only within a closed set, so that a prey species is defined by its strategy $u$, the predator by $v$, and the super-predator by $w$, within the constraints:

$$
u(t) \in U, \quad v(t) \in V, \quad w(t) \in W
$$

where $U, V$, and $W$ are closed sets included in $[0, \infty[$. In this formulation, any predator characterized by $v \in V$ can hunt a prey of strategy $u \in U$, and any super-predator $w \in W$ can hunt a predator $v \in V$. Sih et al (1985: 296) for example concluded that "environmental stress can either increase or decrease the importance of predation", and that "the outcome depends on the relative effects of stress on predation rates and on prey growth rate". Hickman (1975) showed that plants of annual species Polygonum cascadense allocate proportionally more resources to reproduction in harsh open habitats than in more moderate habitats, and that these differences are developmentally plastic, adapting to short-term environmental unpredictability. Giesel (1976: 75) from studies of passerine birds concludes that the variability in fertility 
is an "important adaptation to account for environmentally induced unpredictability of the major selective forces". Wilbur (1972) observes the plastic growth rate and variable size at metamorphosis of amphibians as adaptations to the uncertain environment of temporary ponds. This morphological variation are induced defenses rather than a genetic polymorphism (McCollum, 1993).

The assumption that strategies can vary within certain fixed bounds changes the kind of results to be expected: The quest for asymptotic patterns such as attractors is no longer at stake, we now pay attention to all routes issuing from all states. Among the possible strategies, some are deadly: starting from a given state of food chain, does a trajectory exist in $\left(x_{1}, x_{2}, x_{3}\right)$ remaining in a given set defining coexistence forever (or until a given time horizon)? Among the admissible strategies, only the viable ones are observed in the long term: those which enable the players to stay in the game. A system taking non viable strategies at least once is doomed to leave the set of constraints, and subsequently is led to extinction.

Aubin (1992: 128; 1997: 240) showed how permanence is a specific case involving viability. He considers a closed set $K$ containing a set $\Omega$ such that $\bar{\Omega} \subset \operatorname{Int}(K)$ and

$$
\partial K=\operatorname{Viab}(K \backslash \Omega)
$$

The first condition will open the opportunity of asymptotic states different from extinction. The second condition reflects the fact that the system can no longer escape the boundary $x_{i}=0, i=1,2$ or 3 once it is attained: there is no way to recover the extinct species. Permanence occurs in the special case where $\Omega$ is invariant, that is to say all solutions starting from $\Omega$ remain in $\Omega$. 
What is important is that the boundary is not hit, a property which was simplified into the existence of a $\delta>0$ such that $\delta<\liminf _{t \rightarrow \infty} x_{i}(t):$ the system remains away from extinction with a "security" ring of width $\delta$. Permanence is a more stringent property than strong persistence, which requires $\delta=0$ and persistence which requires $0<\lim \sup _{t \rightarrow \infty} x_{i}(t)$ (Hofbauer and Sigmund, 1988: 161). Indeed, as, generally, lim inf $\leq$ lim sup, permanence implies persistence. However, the concept of limsup is hard to generalize outside the narrow context of the simplex. Having persistence without strong persistence is a very particular concept, meaning that an almost extinct $x_{i}$ will reappear in the future, and was forged notably for the Lotka-Volterra model (Jansen and Sigmund, 1998). It Local stability says that there exists a neighborhood $V_{1}$, such that for all neighborhood $V_{2} \subset V_{1}$, there exists a neighborhood $V_{3}$ such any solution starting from $V_{3}$ returns to $V_{2}$. This is a property also specific of the simplex and hard to generalize.

The viability property, stating how strategies should change at time $t$ so that a qualitative property, here remaining above thresholds, keeps at least one chance to be safeguarded forever, is general to any space and applies to transient systems. The viability conditions are raised at each time, not necessarily in the neighborhood of equilibria or attractors. We have no worry of predicting the future trajectory the system will take; rather we delineate the largest set of states from which there exists at least one route remaining in a given set $K$ forever. This largest set is called the viability kernel and denoted $\operatorname{Viab}_{F}(K)$ (Aubin, 1991). The viability kernel is a set of initial conditions. Extinction is certain for states outside the viability kernel in finite time; from viable states, solutions can also lead to extinction. 
The viability kernel has natural selection at its basis, because it helps making out those states from which there exists at least one possibility for the food chain to continue from those doomed to lose at least one trophic level. To remain in the viability kernel, the species do not really "select" strategies which are viable. The viability kernel simply reveals that, should the population densities be shifted outside of the viability kernel, then there is no way the populations could avoid extinction, however adaptable strategies $u, v$, and $w$ are, however flexible these interactions are at restructuring and hence redefining their strategies within the constraints. Mathematically, the strategies are just measurable, not necessarily continuous, they can change instantaneously within their respective sets of admissible strategies $U, V$, or $W$. If it is impossible for infinitely flexible strategies (capable of varying instantaneously within given bounds) to rescue the situation, then adaptation under the constraints of natural selection can do no better.

We shall reveal the strategies which enable the system to be viable, and show that they are rendered state-dependent feedbacks on the boundary of the viability kernel. This does not at all entail that species would choose some appropriate control to keep the system viable. We just say that species surviving in the long term have had viable strategies, species which went extinct or species which can no longer avoid extinction have taken a non viable strategy. Viability portrays natural selection, without appealing to fitness maximization. A surviving species is no longer of maximal fitness, but has managed to be viable so far, notably in unpredictable environments.

This approach was used in the case of two-species Lotka-Volterra models to show that the coexistence kernel of prey and predator (from each state 
of this set, there exists at least one route along which prey and predator have sufficiently high densities) is the intersection of the viability kernels of prey and of predator (Bonneuil and Müllers, 1997). In the two-species case, the boundaries of the viability kernels were obtained as solutions of specific differential equations. In extending the complexity from two to three dimensions, this method becomes hard to handle, and we resort rather to the clever viability algorithm, never used before in ecosystem dynamics, devised by Saint-Pierre (1994), and presented in appendix.

\section{The Dynamical Game and the Viability Prob- lem}

We consider a food chain made of prey, predator and super-predator of respective densities $x_{1}(t), x_{2}(t)$ and $x_{3}(t)$, in the Lotka-Volterra model of interactions:

$\left\{\begin{array}{ccccc}x_{1}^{\prime}(t) & = & x_{1}(t)\left(1-u(t) x_{1}(t)-v(t) x_{2}(t)\right) & := & f_{1}(x(t), u(t), v(t), w(t)) \\ x_{2}^{\prime}(t) & = & x_{2}(t)\left(-b+\alpha v(t) x_{1}(t)-w(t) x_{3}(t)\right) & := & f_{2}(x(t), u(t), v(t), w(t)) \\ x_{3}^{\prime}(t) & = & x_{3}(t)\left(-c+\beta w(t) x_{2}(t)\right) & := & f_{3}(x(t), u(t), v(t), w(t))\end{array}\right.$

where $x(t)=\left(x_{1}(t), x_{2}(t), x_{3}(t)\right)$ and where $b>0$ and $c>0$ denote rates of mortality due to factors other than predation. Setting the reproductive rate of the prey to one implies that other vital rates are implicitly scaled to this rate. $\alpha$ and $\beta$ are physiological parameters portraying energy transfer between trophic levels, which also govern reproductive rates of the predator 
and super-predator, respectively; and

$$
u(t) \in\left[u_{\min }, u_{\max }\right]=U, \quad v(t) \in\left[v_{\min }, v_{\max }\right]=V, \quad w(t) \in\left[w_{\min }, w_{\max }\right]=W
$$

denote the inverse of the self-limiting capacity of the prey and the predation rates respectively. $u(t)$ describes strategies of intra-specific competition among prey, while $v(t)$ and $w(t)$ describe the predation strategies of the predator and super-predator, respectively, in conjunction with the defense strategies of the prey and predator, respectively.

Two noteworthy examples of $u, v$, and $w$ are Lotka-Volterra three-trophic level food chains obtained with $u(t), v(t)$ and $w(t)$ constant (Rescigno and Jones, 1972); or Holling type 2 interactions corresponding to $u(t)=1, v(t)=$ $a_{1} /\left(1+b_{1} x_{1}(t)\right)$ and $w(t)=a_{2} /\left(1+b_{2} x_{2}(t)\right)$, where the $a_{i}$ s and the $b_{i}$ s combine intrinsic growth rates, maximum predation rates, half-saturation constants, efficiencies of predator or super-predator and the carrying capacity of the prey (Kuznetsov and Rinaldi, 1996). In these two examples, $u, v$ and $w$ do not depend explicitly on time. Here, we avoid to specify $u, v$, and $w$ either as constants or as functions of the states. For a system perpetuating itself, the $u, v$ and $w$ appear as feedbacks from the constraints, those which enable the system to keep at each time at least one possibility to perpetuate itself forever. These strategies are called viable. For those systems going to extinction, the $u, v$ and $w$ take non viable values at least once.

We consider that a species is extinct when the density decreases below some fixed threshold, which, after appropriate re-scaling, we can choose as:

$$
\underline{x}_{1}=1, \quad \underline{x}_{2}=1, \quad \underline{x}_{3}=1
$$


(The re-scaling changes the interpretation of $\frac{1}{u}$, which no longer stands as the carrying capacity of the prey, only with a factor $\frac{1}{\underline{x}_{1}}$.)

System (3) represents a dynamical game, where prey, predator, and superpredator constitute the three players, and where $u(t), v(t)$ and $w(t)$ represent the respective strategies at time $t$. The dynamical game consists in having the system change so that the three species can keep the possibility at each time to survive forever: the strategies chosen by each player-species are not directed toward any pay-off and winning is not an issue: the implied aim of each player is the ability to continue playing the game forever. In mathematical terms, the game consists in safeguarding at least one solution of the system remaining in a fixed closed set forever.

Various dynamical games can be considered, according to the point of view of each protagonist:

- Viability of the prey:

The subset of $K_{1}:=[1, \infty[\times[0, \infty[\times[0, \infty[$ is viable if and only if, for any initial condition $\left(x_{1}(0), x_{2}(0), x_{3}(0)\right)$ of prey, predator and superpredator densities, there exists at least one solution $\left(x_{1}(),. x_{2}(),. x_{3}().\right)$ of Eq. (3) regulated by strategies $u(),. v(),. w($.$) satisfying (4) which$ complies to the constraints:

$$
\text { for all } t \geq 0, x_{1}(t) \geq \underline{x}_{1}=1, x_{2}(t) \geq 0, x_{3}(t) \geq 0
$$

- Viability of the predator:

Similarly, if attention is paid to the predator, only the populations of predator maintaining themselves above the threshold $\underline{y}$ are viable. The set of constraints is thus $K_{2}:=[0, \infty[\times[1, \infty[\times[0, \infty[$; 
- Viability of the super-predator:

The set within which to remain is $K_{3}:=[0, \infty[\times[0, \infty[\times[1, \infty[$;

- coexistence of super-predator, predator and prey: A point $x^{0}=\left(x_{1}^{0}, x_{2}^{0}, x_{3}^{0}\right)$ belongs to the food chain coexistence kernel if there exists at least one solution $x()=.\left(x_{1}(),. x_{2}(),. x_{3}().\right)$ of the dynamical game made of Eq. 3 and 4 starting from $x^{0}$ and satisfying:

$$
\text { for all } t \geq 0, x_{1}(t) \geq \underline{x}_{1}=1, x_{2}(t) \geq \underline{x}_{2}=1, x_{3}(t) \geq \underline{x}_{3}=1
$$

that is to say, remaining in a given set $K$ :

$$
x(t) \in K:=K_{1} \cap K_{2} \cap K_{3}
$$

We study here the coexistence kernel for the three trophic levels. Equations (3) and (4) can synthetically be written under the differential inclusion:

$$
x^{\prime}(t) \in F(x(t))
$$

under constraints

$$
x(t) \in K
$$

where:

$$
\begin{aligned}
F(x(t)):= & \left\{\left(x_{1}(t)\left(1-u(t) x_{1}(t)-v(t) x_{2}(t)\right), x_{2}(t)\left(-b+\alpha v(t) x_{1}(t)-w(t) x_{3}(t)\right),\right.\right. \\
& \left.\left.x_{3}(t)\left(-c+\beta w(t) x_{2}(t)\right)\right) \mid u(t) \in U, v(t) \in V, w(t) \in W\right\} \\
:= & \{f(x, u, v, w) \mid u(t) \in U, v(t) \in V, w(t) \in W\}
\end{aligned}
$$

is a point-to-set map, also called correspondence.

Aubin (1991) showed the existence of the viability kernel of the set $K$, the largest closed viable set in $K$ under the dynamic $F$. 


\section{Results}

\subsection{The viability kernel}

Figure 1 shows the viability kernel for certain values of $U, V$ and $W$. In this example, not all states in $K$ are viable: from these non viable states, the system is doomed to leave $K$ sooner or later. The viability kernel is then smaller than the set of state constraints. Many states outside attractors (notably equilibria) are viable: starting from these states, the food chain can perpetuate itself forever. The section at $x_{3}=1$ is similar to the viability kernel of the system made of prey and predator alone (Bonneuil and Müllers, 1997).

The maintenance forever depends on the selection at each time of viable interactions $u, v$, and $w$ : these viable interactions are defined as those for which there exists at least one route remaining in $K$ forever. These viable interactions are those for which the direction $x^{\prime}(t)$ at $t$ belongs to a specific set, the contingent cone, which extends the concept of tangent space to any closed set.

For any set $M \in x$, the contingent cone $T_{M}(x)$ at state $x$ is defined by:

$$
T_{M}(x)=\left\{\tilde{x} \in \mathbb{R}^{3} ; \liminf _{h \longrightarrow 0+} \frac{1}{h} d_{M}(x+h(\tilde{x}))=0\right\},
$$

where $d_{M}(x)=\inf _{w \in M}\|w\|$. If $M$ is convex, $T_{M}$ coincides with the tangent cone used in convex analysis.

Aubin (1991) showed that if the set $\tilde{K}$ is a viability domain, then it 


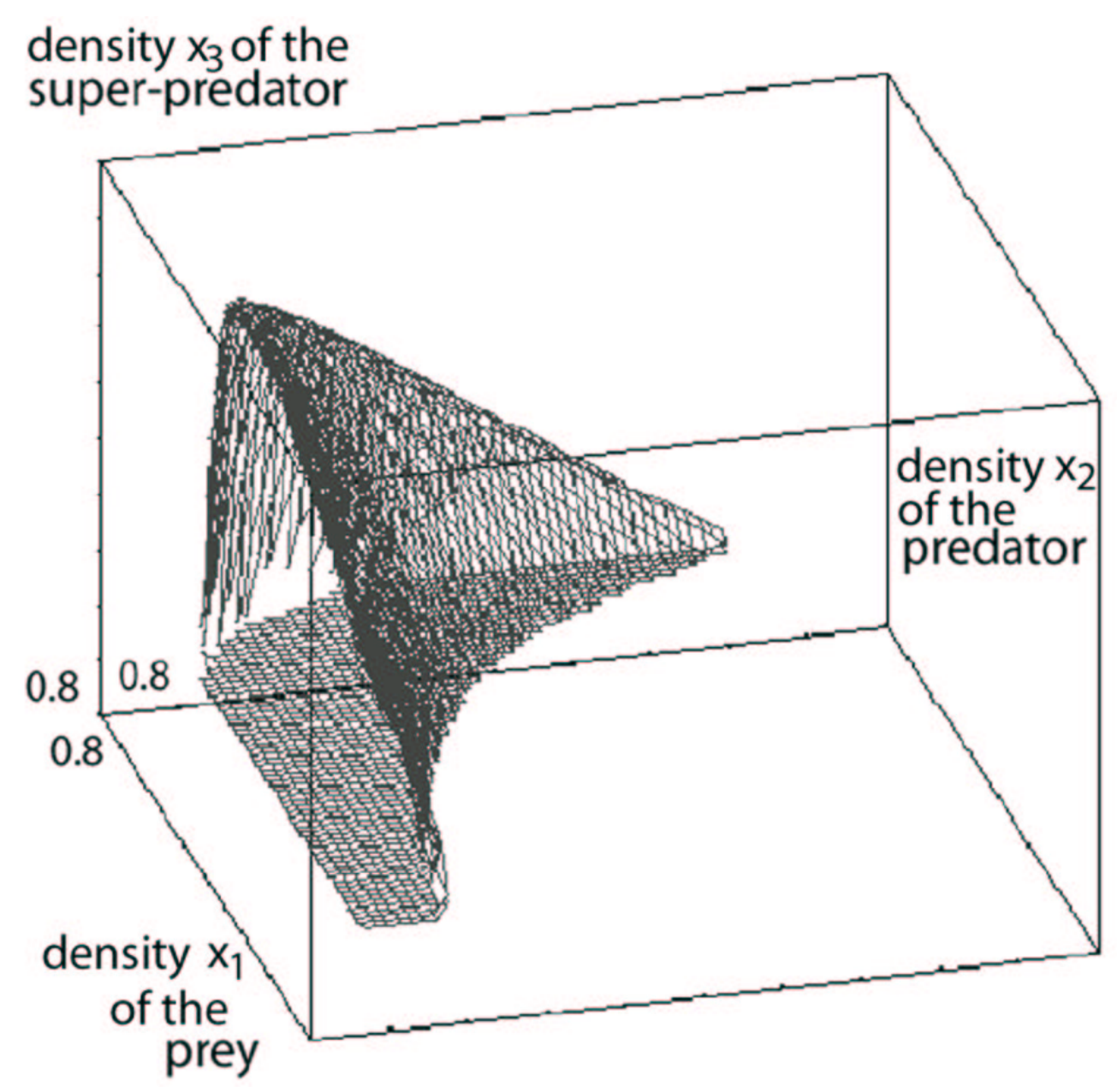

Figure 1: Viability kernel of the three-trophic food chain: $U=[0.20 ; 0.21]$, $V=[0.12 ; 0.13], W=[0.10 ; 0.11]$ : from each state of this set, there exists at least one trajectory remaining in the set of state constraints forever 
satisfies the following tangential condition:

for all $x \in \tilde{K}$ there exists $u \in U, v \in V, w \in W$ such that

$$
\left(f_{1}(x, u, v, w), f_{2}(x, u, v, w), f_{3}(x, u, v, w)\right) \in T_{\tilde{K}}(x) .
$$

This holds also true for $\operatorname{Viab}_{F}(K)$, the largest closed viability domain contained in $K$.

In the interior of the viability kernel, all strategies $u, v, w$ are viable: from each state in the interior, there exists a solution remaining in $K$ forever. The difficulty lies on the boundaries. We computed viable strategies on this boundary. As an example, Figure 2 shows the viable strategy $v(t)$ on the boundary in the direction of high super-predator density of the viability kernel of Figure 1. This strategy appears very irregular, alternating abruptly between $v_{\min }$ and $v_{\max }$. Similar figures are obtained for $u$ and $w$ on the boundary in the direction of high super-predator density. Remaining along this boundary thus demands strategies flexible in time: the system is then fragile and easy to destabilize when the super-predator is important in density. Viable strategies on the lower boundary are smoother though not constant, implying that coexistence is obtained with less variation of the interactions when the super-predator is of low density.

Empirical studies where viable and non viable strategies are estimated are few, because non viable species in nature are doomed to extinction and thus are rare, and because of the evolutionary scale of time, which renders experiments often impractical (Sih et al, 1985).

In experimental studies, Luckinbill (1973) studied predator Didinium nasutum and prey Paramecium aurelia, itself feeding on Aerobacter aerogenes. Attempts at the prolonged laboratory study in these cultures "lacking refuges 


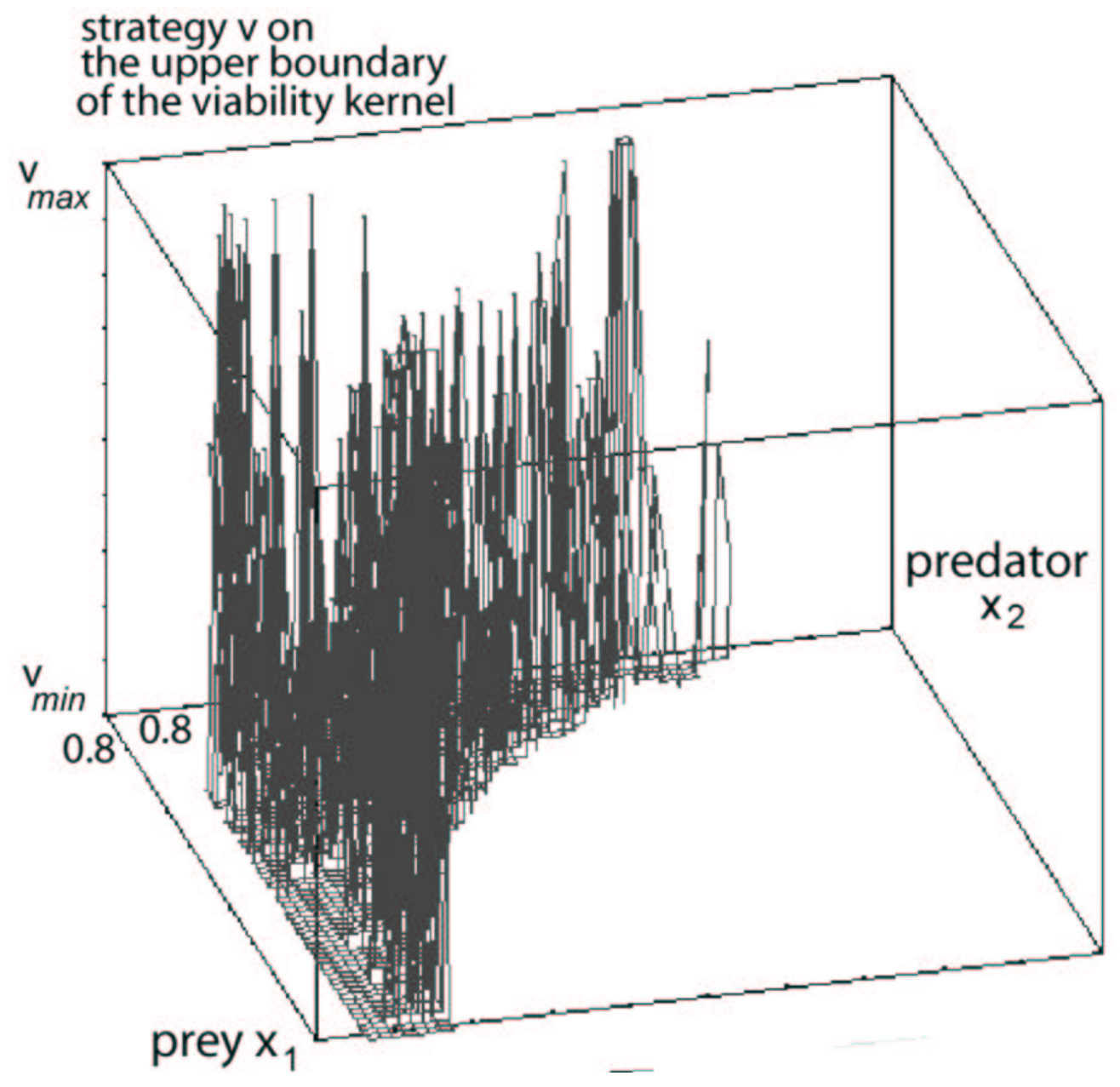

Figure 2: Viable strategy $v(x)$ along the boundary of the viability kernel for $U=[0.20 ; 0.21], V=[0.12 ; 0.13], W=[0.10 ; 0.11]$ : on each state $x$ of the boundary of the viability kernel, having a solution remaining in $K$ forever is possible only if the viable strategy $v(x)$ is used 
or physical complexity" lead to the extinction of the predator. The coexistence is obtained only when he added methyl cellulose into the culture, which has the "important effect of reducing the rate of movement of both $P$. aurelia and D. nasutum without giving the prey a dispersal advantage" (:1322). The ensuing reduced frequency of encounter between predator and prey enhances the ability of both species to coexist, provided that the availability of nutrient is fine-tuned. It is indeed necessary in this experiment "both that predators be unable to capture all the prey in the system and that some restriction of prey growth exist in addition to predation" (:1326). If we view this experiment within the framework of Eq. (3), we understand interactions $u, v$, and $w$ in the absence of methyl cellulose as non viable. Slowing down the movements alters these interactions, and nutrient is adjusted so that the initial state is viable.

Another related case study is offered by Wilbur (1983). He showed that only small tadpoles are killed by predaceous insects, and that rapid growth appears as a defense against such gape-limited predators. Slow growth leads to the complete elimination of tadpoles, unless tadpoles are sufficiently dense to satiate predators. Rapid growth and morphological metamorphoses constitute then viable strategies; a long larval period and the morphology prevailing in the absence of predators become non viable in presence of predators. Moreover, he demonstrates the role of initial conditions and history in determining the trajectory of a community, thus in coherence with the logic of the viability kernel, which is a set of initial conditions. This example of tadpoles is a fascinating case study where the group is on the verge of being totally devored, where initial conditions in tadpoles and newts combine with 
metamorphoses of tadpoles confronted with predations to permit a few individuals to escape from predation. We are in the logic of viable strategies, viable initial states, and viability boundary.

Wilbur (1997: 2293) varied again initial conditions in an array of 144 experimental ponds in which he could follow the course of the community structure throughout a season. He observed that newly migrated predators "can reduce, even eliminate, prey, and that these risks change with relative body sizes of predator and prey" (: 2280). Individuals decrease active foraging or develop morphological adaptations, such as changes in coloration and shape of tails. Tadpoles with wider tails swim faster to escape predator Anax (: 2293), but in the absence of predator, have a higher mortality than tadpoles with narrower tails. Coloration or the width of the tail stand for viable strategies, while narrow tails and normal color become non viable in the presence of predators.

\subsection{Dependence of the Viability Kernel on Admissible Strategies and Destabilization}

The viability kernel increases with the sets $U, V$, and $W$ of admissible interactions, as Table 1 shows.

Its volume is empty when $U, V$ or $W$ are narrow enough, then increases rapidly in a logistic manner to reach an upper limit, equal to the set $K$ itself. Figure 3 shows how the volume of the viability kernel increases with the most influencing bounds, $v_{\min }$ describing the predator, and $w_{\min }$ from the super-predator. Notably, it emphasizes that the coexistence of the three species needs that the super-predator must repeatedly take a sufficiently low 


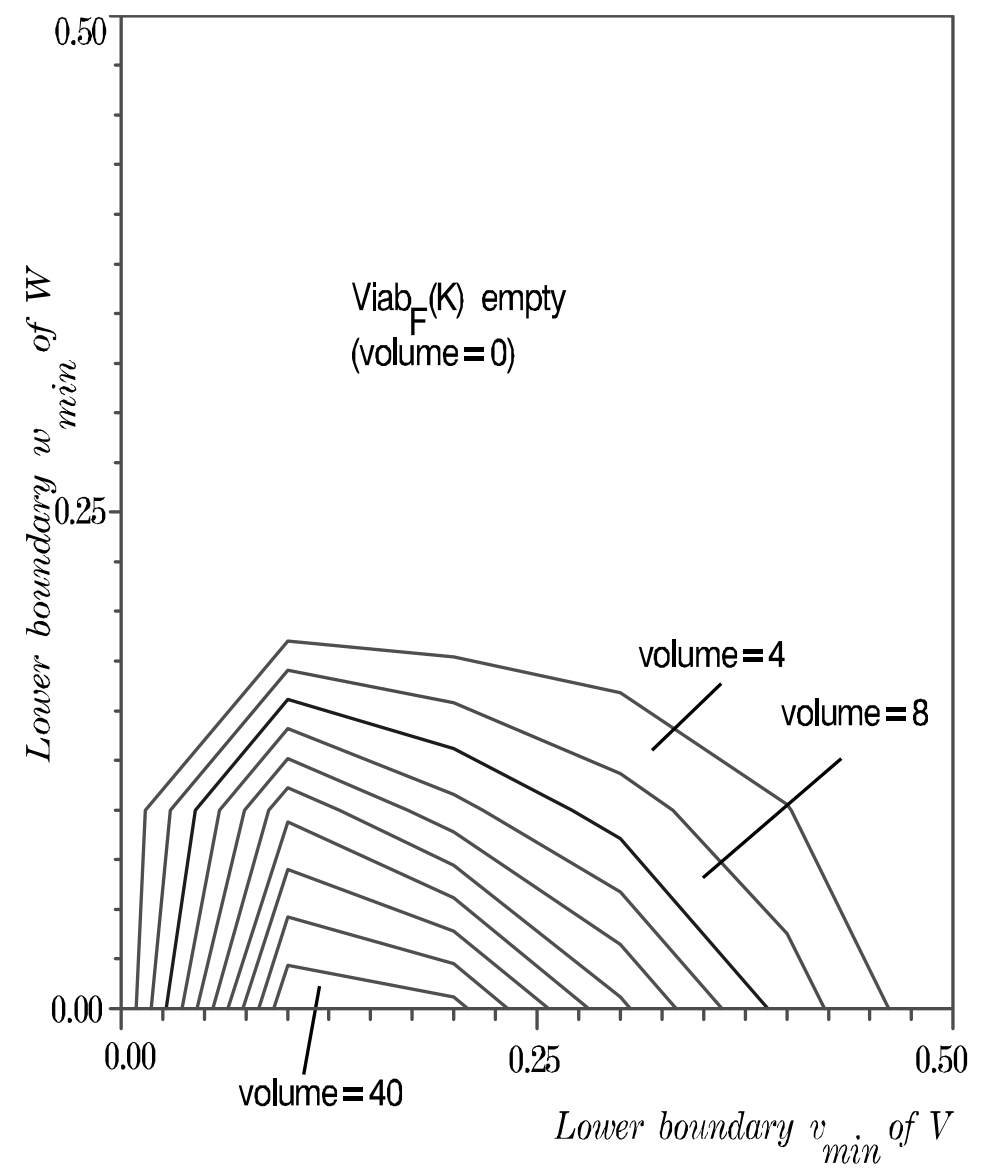

Figure 3: Variation of the volume of the viability kernel in $v_{\min }$ and $w_{\min }$, when $u_{\min }, v_{\min }, w_{\min }, u_{\max }, v_{\max }, w_{\max }$ are varied over $[0,0.5]$ by steps of 0.01 
Table 1: Linear effects in the regression of the volume of the viability kernel on the bounds of the set of admissible strategies $U, V$ and $W$

\begin{tabular}{ccc}
\hline & Coefficient & std \\
\hline Intercept & 12.44 & 0.67 \\
$u_{\min }$ & -12.03 & 1.41 \\
$u_{\max }$ & 19.40 & 1.29 \\
$v_{\min }$ & -40.48 & 1.53 \\
$v_{\max }$ & 9.80 & 1.51 \\
$w_{\min }$ & -51.73 & 1.16 \\
$w_{\max }$ & 16.97 & 1.16 \\
\hline
\end{tabular}

capture rate $w$, and that the predator cannot have too high its own strategy $v$. This stands in accordance with more classical theory on asymptotic time horizons that predator prey systems are endangered when predators are too effective, devoring prey far below the capacity of the prey to restock the milieu. The repetition of sufficiently low values for $v$ does not have to be cyclic, it depends on where the system travels within the viability kernel (if it remains viable). The search for cyclic patterns in empirical systems should then be superseded by the search of mere repetitions.

One piece of news is that the set of the states where the prey is endangered by too efficient predators, is contained in the viability boundary. The states where the prey can no longer restock the milieu, how flexible its own strategy, are outside the viability kernel. The viability boundary can contain equilibria, as in classical theory. On the viability boundary, which is a twodimensional manifold, the set of viable strategies $u, v$ and $w$ permitting the 
possibility of coexistence now and in the future depends on the state $X$, thus letting these viable strategies appear as state-dependent "feedbacks" $u(X)$, $v(X)$ and $w(X)$ obtained by the very construction of the viability boundary (instead of a dependence on $X$ a priori). Either the system remains on the boundary forever or it leaves this boundary to go extinct in finite time. In the interior of the viability kernel, when the state is not on the boundary, any strategy within the fixed bounds can lead to a viable solution: in this sense, the strategy is not determined as a feedback yet. The notion of feedback is linked to that which happens on the boundary.

Wilbur (1997: 2293) notes that tadpoles with thin tails survive if their density is high enough so as to satiate newts: the system remains in the interior of the coexistence kernel and there is no need to change interactions. When tadpole density collapses, metamorphoses in color, the acceleration of growth (shortening of the larval period) and the widening of the tail, even at the cost of a higher mortality, permits the survival of the biggest tadpoles, which are few. Although these experiments were not specifically designed to reveal viability kernels, they however offer a striking example of a prey changing sufficiently rapidly its interactions with its predators so as to render them viable.

Many results on the effect of increasing the concentration of nutrient input apply to equilibria, which can be destabilized (Luckinbill, 1973; Morin et Lawlor, 1995; Boer, Kooi, and Kooijman, 2001). Neill (1988) found that adding nutrients to enclosures in an oligotrophic lake improved rotifer recruitment which supported high densities of midge larvae (Chaoborus), which in turn overexploited their alternate crustacean prey. In a study conducted in 
estuarine enclosures, Björnsen et al (1988) showed that correlated oscillations in bacteria and heterotrophic flagellates increased in amplitude when nutrients were added.

Equilibria within the set of constraints by definition belong to the viability kernel. The viability kernel also contains all transient states from which coexistence is possible forever: adding nutrients sufficiently shifts the initial state out of the viability kernel, where the interactions $u(t), v(t)$ and $w(t)$ are no longer flexible enough to restore the possibility to remain in the set of constraints forever. The viability kernel thus permits to address the extinction of prey or predators resulting from oscillations of very high amplitude, as they tend to be in enriched systems, and help identify viable strategies when the system is prone to high amplitude oscillations. If the disturbance is not of high enough amplitude, and the system remains in the viability kernel, the species continue to coexist (such as in McCauley and Murdoch, 1990, studying Daphnia feeding on algae). A sufficient exogenous addition of predator (or of super-predator) can push the state out of the viability kernel, implying by definition the destruction of the system before long. Morin and Lawler (1995) noticed extinction when the predators Didinium and Woodruffia are added in sufficient quantity, but these predators can sometimes coexist with prey for long periods under other conditions (Luckinbill, 1973, 1974; Salt, 1967).

This different response depending on the quantities of predators added and on other conditions enters again into the very principle forecast by the concept of the viability kernel, which delineates the states from which a trajectory exists with coexistence and those from which there exists no such 
trajectory. Figure 4 shows a trajectory starting from a viable state $B$ : it remains within $K$ and converges in this case to an equilibrium. Shifting the density of prey $x$ so that the new initial state $A$ is outside the viability kernel, there is no longer any trajectory remaining within $K$ (defined in Eq. 8). Figure 4 shows an arbitrary solution starting from $A$ : while rolling around, it leaves $K$, driving the prey to attain too small densities.

The same holds true for the predator: shifting the predator density $y$ to too high a value, so that the starting state is outside of the viability kernel, destabilizes the system which will violate the constraints. Symmetrically, starting from too low a density in predator has the same effect, because of the shape of the viability kernel represented on Figure 1, leaving states with low values of prey and predator non viable. In this case, adding predator stabilizes the system in rendering the new initial state viable. Concerning the predator, this property of the viability kernel complies with Mittelbach et al (1995) who showed that the elimination and reintroduction of a top predator in Wintergreen Lake dramatically changed the species composition and size structure of the fish and zooplankton communities.

The shape of the viability kernel on Figure 1 also implies that this property of stabilization or destabilization does not hold for the super-predator: adding super-predator does not threaten the conservation of the three species as long as the initial state after the addition of super-predator still lies within the viability kernel. This complies with Morin and Lawler (1995) who found no consistent effects of omnivores on prey dynamics for two omnivore species feeding on two different prey. For too high an addition, the initial state is shifted outside of the viability kernel and the system is then doomed to cross 


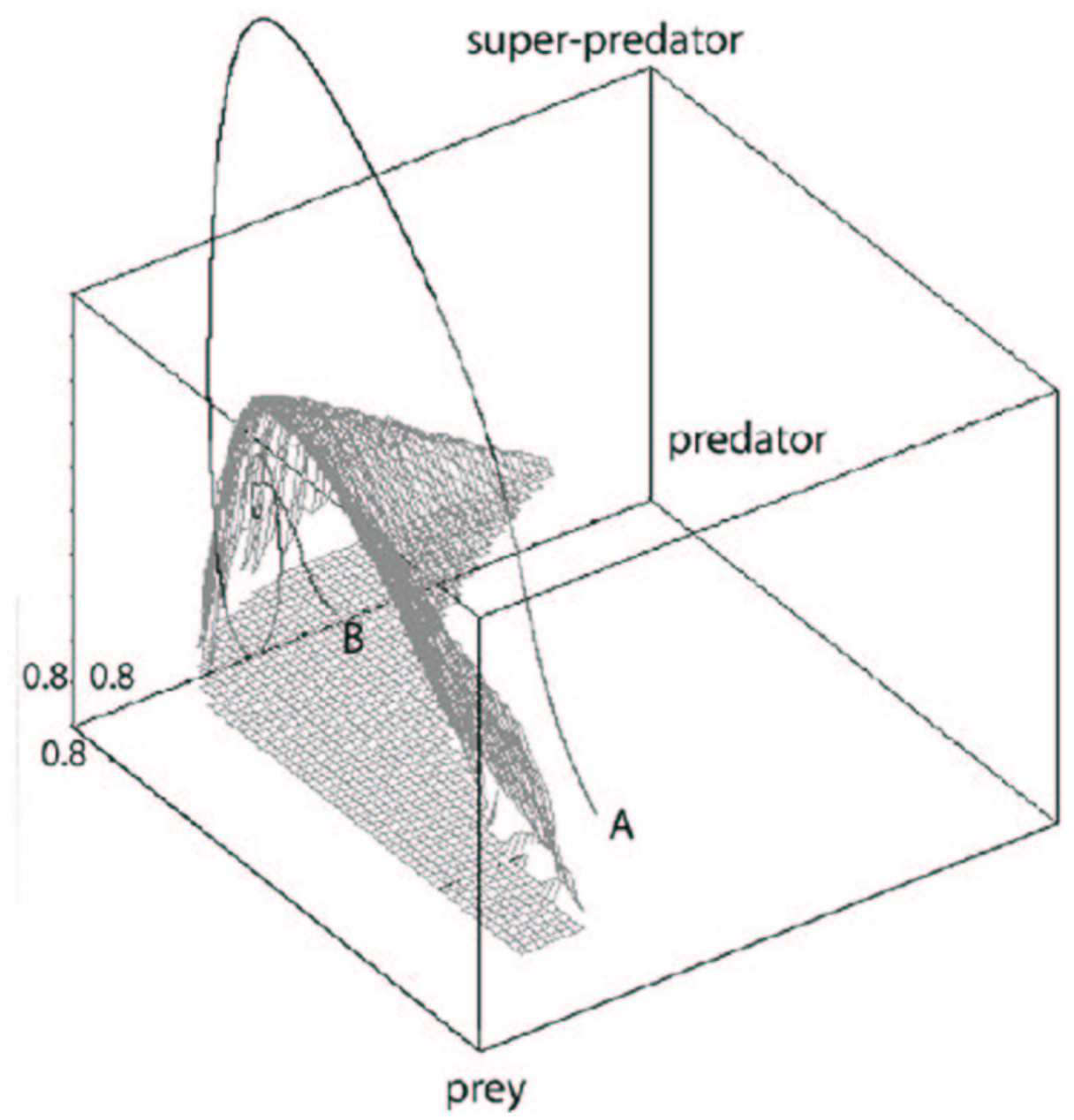

Figure 4: Example of a trajectory starting from viable state $B$. After a shift in prey density, no trajectory can ever maintain the prey over the threshold density. Such a trajectory starts from the non viable state $A$ 
the constraints. Varying the capture rate $w$ of the super-predator alters the kernel, but does not change the projection onto the prey-predator plane significantly: there is no prey-predator non viable state rendered viable by the addition of super-predator. Muratori and Rinaldi (1992) showed theoretically that Holling type interactions permit that the addition of a suitable superpredator with linear functional response to a standard prey-predator system characterized by stable limit cycle can give rise to a food chain with a unique positive stable equilibrium. However, this theoretical stabilizing effect does not account for the violation or not of the constraints during the process. The consideration of constraints also during the transient trajectories is a plus brought by viability theory.

\subsection{Viability of a Single Species}

\section{Conclusion}

Lawton (1989) pointed out that predictions of food web theory are relatively bad on natural populations. We precisely acknowledged the unpredictability of environment through $u, v$, and $w$ varying in an unpredictable manner within closed sets, and suggested instead that the concept of viability precisely answers the question of the maintenance of the ecosystem within density thresholds of transient solutions, not restricted to what occurs in the neighborhoods of equilibria and attractors.

Morin and Lawler (1995) called for a better understanding of the organizing forces in ecosystems: the bare mathematical translation of having the three species remaining within closed sets brings an organizing princi- 
ple stemming from the confrontation of intrinsic dynamics and constraints. Nagumo (1942) pioneered the mathematics of remaining within a closed set $K$ in the case of a differential equation. The addition of set-valued analysis pioneered by Aubin (1991) permits to take into account the variability and unpredictability inherent in ecosystem dynamics, and the concept of viability kernel permits to delineate specific states, the viable states, answering the question of the perpetuation of the ecosystem.

That is a reason why we hope and tried to show that this tool would bring some useful insights into ecosystem dynamics.

\section{References}

1. Abrams, P.A.. 1987. Alternate models of character displacement. I. Displacement when there is competition for nutritionally essential resources. Evolution 41: 651-661.

2. Aubin, J.-P., 1992. Viability Theory, Birkhäuser, Boston.

3. Aubin, J.-P., 1997. Dynamic EConomic Theory, Springer, Berlin.

4. Björsen, P. K., B. Riemann, S.J. Horsted, T.G. Neilson and J. PockSten. 1988. Trophic interactions between heterotrophic nanoflagellates and bacterioplankton in manipulated seawater enclosures. Limnol. Oceanogr. 33: 409-20.

5. Boer, M. P., B. W. Kooi and S. A. L. M. Kooijman,. 2001. Multiple attractors and boundary crises in a tri-trophic food chain, Mathematical Biosciences 169: 109-128. 
6. Bonneuil, N. and K. Müllers. 1997. Viable populations in prey-predator systems, J. Math. Biol. 35: 261-293.

7. Brown, J. S., T. L. Vincent. 1985. The evolutionary game, Theoretical Population Biology 31: 140-166.

8. Brown, J. S., T. L. Vincent. 1987. Coevolution as an evolutionary game, Evolution 41-1: 66-79.

9. Brown, J. S. and Vincent, T. L. 1992. Organization as an evolutionary game, Evolution, 46-5: 1269-1283.

10. Chiu, C-H and Hsu S-B. 1988. Extinction of top-predator in a threelevel food-chain model, Journal of Mathematical Biology 37: 372-380.

11. Chesson, P. L. 1985. Coexistence of competitors inspatially and temporally varying environments: a look at the combined effects of different sorts of variability, Theoretical Population Biology 28: 263-287.

12. Drake, J. A. 1990. The mechanics of community assembly and succession, Journal of Theoretical Biology 147: 213-233.

13. El-Owaidy H. and A. A. Ammar. 1986. Mathematical Analysis of a food-web model, Mathematical Bioscience 81: 231-237.

14. Frankowska, H. and M. Quincampoix. 1991. Viability kernels of dynamical games with constraints: algorithm and applications, Journal of Mathematical Systems, Estimation and Control 1: 371-388.

15. Freedman, H. I. and J. W. H. So. 1985. Global stability and persistence of simple food chains, Mathematical Biosciences 76: 69-86. 
16. Freedman, H. I. and P. Waltman. 1977. Mathematical Analysis of some three species food-chain models, Mathematical Bioscience 33: 257-276.

17. Freedman, H. I. and P. Waltman. 1984. Persistence in models of three interacting predator-prey populations, Mathematical Bioscience 68: 213-231.

18. Gard, T. C. 1980. Persistence in food webs: Holling-type food chains, Mathematical Bioscience 49: 61-67.

19. Gard, T. C. and T.G. Hallam. 1979. Persistence in food-webs-I, LotkaVolterra food chains Bulletin of Mathematical Biology 41 877-891.

20. Giesel, J. T. 1976. Reproductive strategies as adaptations to life in temporally heterogeneous environments, Ann. Rev. Ecol. Syst. 7: $57-79$.

21. Gutierrez A. P., Mills N. J., Schreiber S. J. and Ellis, C. K. 1994. A physiological based tritrophic perspective on bottom-up-top-down regulation of populations, Ecology 75-8: 2227-2242.

22. Hastings, A. 1988. Food web theory and stability. Ecology 69: 16651668 .

23. Hastings, A. and Powell T. 1991. Chaos in a three-species food chain. Ecology 72: 896-903.

24. Hickman, J. C. 1975. Environmental unpredictability and plastic energy allocation strategies in the annual Polygonum cascadense (Polygonaceae), J. Ecol. 63: 681-701 
25. Hofbauer, J. and K. Sigmund. 1988. The theory of evolution and dynamical systems. Cambridge University Press, Cambridge.

26. Hofbauer, J. and K. Sigmund. 1998. Evolutionary Games and Population Dynamics, Cambridge University Press, Cambridge.

27. Holling, C. S. 1965. The functional response of predators to prey density and its role in mimicry and population regulation, Mam. Entomol. Soc. Can. 45: 5-60.

28. Huffaker, C. B. 1958. Experimental studies on predation: dispersion factors and predator-prey oscillations. Hilgardia 27: 343-83.

29. Husson, V., R. Law. 1992. Permanence and the dynamics of biological systems, Mathematical Bioscience 111: 1-71.

30. Jansen, V. A. A. and K. Sigmund. 1998. Shaken not stirred: on permanence in ecological communities, Theoretical Population Biology 54, 195-201.

31. Kuznetsov, Y. A. and S. Rinaldi. 1996. Remarks on food chain dynamics, Mathematical Bioscience 134: 1-34.

32. Law, R. and J.C. Blackford. 1992. Self-assembling food webs: a global viewpoint of coexistence of species in Lotka-Volterra communities, Ecology 73-2: 567-578.

33. Law, R., D. Morton. 1996. Permanence and the assembly of ecological communities, Ecology 77-3: 762-775. 
34. Lawton, J. H. 1989. Food webs, in Ecological Concepts, ed J.M. Cherrett, 43-78. Oxford: Blackwell Sci.

35. Luckinbill, L.S. 1973. Coexistence in laboratory populations of Paramecium aurelia and its predator Didinium nasutum. Ecology 54: 1320-27.

36. Luckinbill, L.S.. 1974. The effects of space and enrichment on a predator-prey system. Ecology 60: 1098-102.

37. McCauley, E., W. W. Murdoch. 1990. Predator-prey dynamics in environments rich and poor in nutrients, Nature 343: 455-57.

38. McCollum, S. A. 1993. Ecological consequences of predation-induced polyphenism in larval hylid frogs. Dissertation. Duke University, Durham, North Carolina, USA.

39. Mitchell, P., Arthur, W., and Farrow, M.. 1992. An investigation of population limitation using factorial experiments, Journal of Animal Ecology 61: 591-98.

40. Mittelbach, G., A. Turner, D. Hall, J. Rettig and C. Osenberg. 1995. Perturbation and resilience: a long-term, whole-lake study of predator extinction and reintroduction, Ecology 76-8, 2347-2360.

41. Morin, P. J. and S. P. Lawler. 1995. Food web architecture and population dynamics: Theory and empirical evidence, Ann. Rev. Ecol. Syst. 26: 505-529.

42. Muratori, S. and S. Rinaldi. 1992. Low- and high-frequency oscillations in three-dimensional food chain systems, SIAM Journal of Applied 
Mathematics 52-6: 1688-1706.

43. Nagumo, M. 1942. Über die Lage der integralkurven gewöhnlicher Differentialgleichungen, Proc. Phys. Math. Soc. Japan 24: 551-559.

44. Neill, W.E. 1988. Complex interactions in oligotrophic lake food webs: responses to nutrient enrichment. In Complex Interactions in Lake Communities, ed. SR Carpenter, 31-44. Berlin: Springer-Verlag.

45. Pianka, E. R. 1978. Evolutionary Ecology. Harper and Row Publishers.

46. Pimentel, D., Nagel, W.P. and Madden, J.L. 1963. Space-time structure of the environment and the survival of parasite-host systems, Am. Nat. 97: 141-67.

47. Pimm, S. L. 1991. The balance of nature? Ecological issues in the conservation of species and communities, University of Chicago Press, Chicago, Illinois, USA.

48. Post, W. M. and S. L. Pimm. 1992. Community assembly and food web stability, Mathematical Bioscience 64: 169-192.

49. Quincampoix, M. 1990. Frontières de domaine d'invariance et de viabiliité pour des inclusions différentielles avec contraintes, CRAS série 1 Paris t311: 904-914.

50. Rescigno A. and Jones, K.G., 1972. The struggle for life: III. A predator-prey chain. Bull. Math. Bioph. 34: 521-532.

51. Saint-Pierre, P. 1994. Approximation of the viability kernel, Applied Mathematics and Optimisation 29: 187-209. 
52. Salt, G. W. 1967. Predation in an experimental protozoan population Woodruffia-Paramecium Ecological Monographs 37: 113-44.

53. Scheffer, M.. 1992. Fish and nutrients interplay determines algal biomass. Oikos 62: 271-282.

54. Sih A., P. Crowley, M. McPeek, J. Petranka, and K. Strohmeier. 1985. Predation, competition, and prey communities: a review of field experiments, Ann. Rev. Ecol. Syst. 16: 269-311.

55. Tsuchiya, H. M., J. F. Drake, J. L. Jost and A. G. Fredrickson. 1972. Predator-prey interactions of Dictyostelium discoideum and Escherichia coli in continuous culture. Journal of Bacteriology 110: 1147-53.

56. Vincent, T. L. and J. S. Brown. 1986. Evolution under nonequilibrium dynamics, In Math. Modelling in Science and Technology, Proceedings of the Fifth ICMM 29-31-8: 766-771.

57. Vincent, T. L. and J. S. Brown. 1989. The evolutionary response of systems to a changing environment, Applied mathematics and computation 32-(2-3): 185-206.

58. Wilbur, H. M. 1997. Experimental ecology of food webs: complex systems in temporary ponds, Ecology 78(8), 2279-2302.

59. Wilbur, H. M. 1987. Regulation of structure in complex systems: experimental temporary pond communities. Ecology 68, 1437-1452.

60. Wilbur, H. M. 1983. Salamander predation and the structure of experimental communities: anuran responses. Ecology 64(6), 1423-1429. 
61. Wilbur, H. M. 1972. Competition, predation, and the structure of the Ambystoma-Rana sylvatica community. Ecology 53, 3-21.

62. Wilder, J. W., Voorhis, N., Colbert, J. J. and Sharov, A. 1994. A three variable differential equation model for gypsy moth population dynamics, Ecological Modelling 72: 229-250.

63. Williamson, M. 1987. Are communities ever stable? in A. J. Gray, M. J. Crawley and P. J. Edwards editors. Colonization, succession and stability. Blackwell Scientific, Oxford: 353-371.

\section{Appendix: The Viability Algorithm (Frankowska and Quincampoix, 1991; Saint-Pierre, 1994)}

Appendix A: characterization of the dynamics $F$

The question :'when can we say that $\forall t \geq 0 x(t) \in K$ ?' can be correctly addressed when $F$ is convex, upper semi-continuous with compact images and $K$ compact.

- The image $F(x)$ is bounded, and, as the dimension of the state space is finite, it is also compact.

- The convexity of $F(x)$ results from its linearity in $u, v$, and $w$.

- How regular is the process? Do small variations on $x(t)$ result in large variations on $x(t+d t)$ ? This property is not necessary to apply viability theorems, but it is useful to run the numerical viability algorithm. For 
any fixed $u, v, w$, does it exist $l \in[0,1]$ such that:

$$
\forall x, y \in K, \sup _{(u, v, w) \in U \times V \times W}|f(x, u, v, w)-f(y, u, v, w)| \leq l|x-y|
$$

which implies the Lipschitz property:

$$
F(x) \subset F(y)+l\|x-y\|
$$

Such a value exists because $f$ is differentiable with respect to $x \in X$ at fixed $u, v, w$, and the components of $\frac{\partial f}{\partial x_{i}}$, for $i=1,2,3$ respectively are bounded on K. The Lipschitz property of $F$ on $K$ implies that $F$ is continuous (a fortiori upper semi-continuous).

$F$ has thus the required property of being Lipschitz, and $K$ is obviously compact. We can thus define the numerical approximation of the discretetime viability kernel.

Appendix B: numerical approximation of the discrete-time $C$-viability kernel

The difficulty is to represent $F(x)$ on a convenient grid of the state space. This question has been addressed by Saint-Pierre (1994), whose algorithm makes it possible to compute viability kernels.

When the correspondence is Lipschitz on $K$, Saint-Pierre considered the approximation $F_{\varepsilon}$ of the correspondence $F$ :

$$
F_{\varepsilon}(x):=F(x)+\varepsilon M(x) \ell(x) \mathcal{B}
$$

where $\mathcal{B}$ is the unit ball, $M(x):=\sup _{y \in F(z), z \in x+\mathcal{B}}\|y\|$ is an estimation of a local bound of the correspondence, $\ell(x)$ is the Lipschitz constant of $F$ in a neighborhood of $x$, and $\mathcal{B}$ is the unit ball of $X$.

Consider a grid $X_{h}$ of $X$ associated with any $h>0$ such that: 
i) the set $X_{h}$ has a finite intersection with any compact of $X$;

ii) $\forall h>0, \forall x \in X, \exists x_{h} \in X_{h},\left\|x-x_{h}\right\| \leq h$

and the projection of the correspondence $F_{\epsilon}(x)$ onto $X_{h}$, defined by:

$$
F_{\epsilon, h}\left(x_{h}\right)=F_{\epsilon}\left(x_{h}\right) \cap X_{h}
$$

Then, for a sequence $\left(x_{h}^{(n)}\right)_{n \in N}$ on $X_{h}$, the initial dynamic (9) becomes the approximated discrete system (Saint-Pierre, 1994):

$$
x_{h}^{(n+1)} \in F_{\epsilon, h}\left(x_{h}^{(n)}\right)
$$

For

$$
K_{h}=(K+h \mathcal{B}) \cap X_{h}
$$

the discrete viability kernel ${ }^{2} \overrightarrow{\mathrm{Viab}}_{F_{\epsilon, h}}\left(K_{h}\right)$ is the set of initial values $x_{h}^{0} \in K_{h}$ of a solution $\left(x_{h}^{(n)}\right)_{n \in N}$ of (17) satisfying $x_{h}^{(n)} \in K_{h}$ for all $n \in \mathbb{N}$. To calculate $\overrightarrow{\mathrm{Viab}}_{F_{\epsilon, h}}\left(K_{h}\right)$, the sequence of sets $K_{\epsilon, h}^{(n)}$ is built according to the algorithm:

$$
\begin{cases}K_{\epsilon, h}^{0} & :=K_{h} \\ K_{\epsilon, h}^{(n+1)} & :=\left\{x_{h} \in K_{\epsilon, h}^{(n)} \text { suchthat } F_{\epsilon, h}\left(x_{h}\right) \cap K_{\epsilon, h}^{(n)} \neq \emptyset\right\}\end{cases}
$$

Saint-Pierre (1994) showed that if $F$ is a Lipschitz correspondence, with compact images, then:

$$
\operatorname{Lim}_{n \rightarrow \infty} K_{\epsilon, h}^{(n)}=\overrightarrow{\operatorname{Viab}}_{F_{\epsilon, h}}\left(K_{h}\right)
$$

and

$$
\operatorname{Lim}_{\epsilon \longrightarrow 0+, \frac{h}{\epsilon} \longrightarrow 0+} \overrightarrow{\operatorname{Viab}}_{F_{\epsilon, h}}\left(K_{h}\right)=\overrightarrow{\operatorname{Viab}_{F}}(K)
$$

\footnotetext{
${ }^{2}$ The arrow over $\overrightarrow{\mathrm{Viab}}_{F_{\epsilon, h}}\left(K_{h}\right)$ emphasizes the discrete nature of the construction. This set is the set of points from which there exists a solution $\left(x_{h}^{(n)}\right)_{n \in N}$ remaining in $K_{h}$.
} 